\title{
Partitioning Orthogonal Polygons by Extension of All Edges Incident to Reflex Vertices: lower and upper bounds on the number of pieces *
}

\author{
António Leslie Bajuelos ${ }^{\mathrm{a}}$, Ana Paula Tomás ${ }^{*, \mathrm{~b}}$ and Fábio Marques ${ }^{\mathrm{c}}$

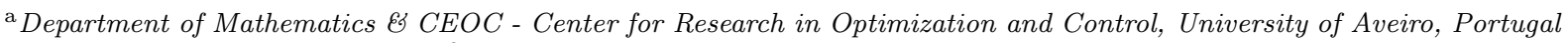 \\ ${ }^{\mathrm{b}} \mathrm{DCC}-\mathrm{FC}$ \& $\mathrm{LIACC}$, University of Porto, Portugal \\ ${ }^{\mathrm{c}}$ School of Technology and Management, University of Aveiro, Portugal
}

\begin{abstract}
Given an orthogonal polygon $P$, let $|\Pi(P)|$ be the number of rectangles that result when we partition $P$ by extending the edges incident to reflex vertices towards $\operatorname{INT}(P)$. In $[4]$ we showed that $|\Pi(P)| \leq 1+r+r^{2}$, where $r$ is the number of reflex vertices of $P$. We shall now give sharper bounds both for $\max _{P}|\Pi(P)|$ and $\min _{P}|\Pi(P)|$. Moreover, we characterize the structure of orthogonal polygons in general position for which these new bounds are exact.
\end{abstract}

Key words: Orthogonal Polygons, Decomposition, Rectilinear Cut, Square Grid.

\section{Introduction}

We shall call simple polygon $P$ a region of a plane enclosed by a finite collection of straight line segments forming a simple cycle. This paper deals only with simple polygons, so that we call them just polygons, in the sequel. We will denote the interior of the polygon $P$ by $\operatorname{INT}(P)$ and the boundary by $\operatorname{BND}(P)$. The boundary shall be considered part of the polygon, that is $P=\operatorname{INT}(P) \cup \operatorname{BND}(P)$. A vertex is called convex if the interior angle between its two incident edges is at most $\pi$; otherwise it is called reflex (or concave). We use $r$ to represent the number of reflex vertices of $P$. A polygon is called orthogonal (or rectilinear) iff its edges meet at right angles. O'Rourke [2] has shown that $n=2 r+4$ for every $n$-vertex orthogonal polygon $(n$-ogon, for short).

\footnotetext{
ऋ (Extended abstract) Partially funded by LIACC through Programa de Financiamento Plurianual, Fundação para a Ciência e Tecnologia (FCT) and Programa POSI, and by CEOC (Univ. of Aveiro) through Programa POCTI, FCT, co-financed by EC fund FEDER.

* Corresponding author

Email addresses: leslie@mat.ua.pt (António Leslie Bajuelos ), apt@ncc.up.pt (Ana Paula Tomás ), fabio@estga.ua.pt (Fábio Marques).
}

Definition 1 A rectilinear cut of an n-ogon $P$ is obtained by extending each edge incident to a reflex vertex of $P$ towards $\operatorname{INT}(P)$ until it hits $\operatorname{BND}(P)$. We denote this partition by $\Pi(P)$ and the number of its elements (pieces) by $|\Pi(P)|$. Each piece is a rectangle, so that we call it $\mathrm{r}$-piece.

Generic $n$-ogons may be obtained from a particular kind of $n$-ogons - the so-called grid orthogonal polygons [3], as illustrated in Fig. 1 (The reader may skip Definition 2 and Lemmas 3 and 4 if he/she has already read [3].)
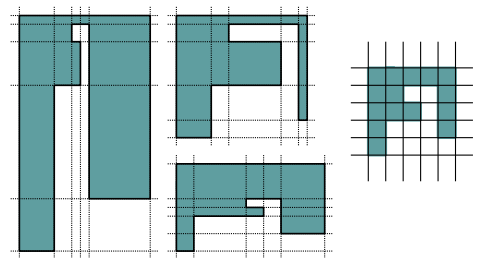

Fig. 1. Three 12-ogons mapped to the same grid 12-ogon.

Definition 2 An n-ogon $P$ is in general position iff $P$ has no collinear edges. We call "grid n-ogon" each $n$-ogon in general position defined in a $\frac{n}{2} \times \frac{n}{2}$ square grid.

Lemma 3 follows immediately from this definition. Lemma 3 Each grid n-ogon has exactly one edge in every line of the grid. 


\section{0th European Workshop on Computational Geometry}

Each $n$-ogon not in general position may be mapped to an $n$-ogon in general position by $\epsilon$-perturbations, for a sufficiently small constant $\epsilon>0$. Consequently, we shall first address $n$-ogons in general position.

Lemma 4 Each $n$-ogon in general position is mapped to a unique grid $n$-ogon through top-tobottom and left-to-right sweeping. And, reciprocally, given a grid $n$-ogon we may create an n-ogon that is an instance of its class by randomly spacing the grid lines in such a way that their relative order is kept.

The number of classes may be further reduced if we group grid $n$-ogons that are symmetrically equivalent. In this way, the grid $n$-ogons in Fig. 2 represent the same class.

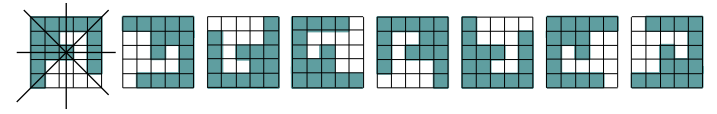

Fig. 2. Eight grid $n$-ogons that are symmetrically equivalent. From left to right, we see images by clockwise rotations of $90^{\circ}, 180^{\circ}$ and $270^{\circ}$, by flips wrt horizontal and vertical axes and flips wrt positive and negative diagonals.

Definition 5 Given an n-ogon $P$ in general position, $G R I D(P)$ denotes any grid n-ogon in the class that contains the grid n-ogon to which $P$ is mapped by the sweep procedure described in Lemma 4

The following result is a trivial consequence of the definition of $\operatorname{GRID}(P)$.

Lemma 6 For all $n$-ogons $P$ in general position, $|\Pi(P)|=|\Pi(G R I D(P))|$.

\section{Lower and Upper bounds on $|\Pi(P)|$}

In [4] we showed that $\Pi(P)$ has at most $1+r+r^{2}$ pieces. Later we noted that this upper bound is not sufficiently tightened. Actually, for small values of $r$, namely $r=3,4,5,6,7$, we experimentally found that the difference between $1+r+r^{2}$ and $\max |\Pi(P)|$ was $1,2,4,6$ and 9 , respectively.

Definition 7 A grid n-ogon $Q$ is called FAT iff $|\Pi(Q)| \geq|\Pi(P)|$, for all grid $n$-ogons $P$. Similarly, a grid n-ogon $Q$ is called THIN iff $|\Pi(Q)| \leq|\Pi(P)|$, for all grid $n$-ogons $P$.

The experimental results supported our conjecture that there was a single FAT $n$-ogon (except for symmetries of the grid) and that it had the form illustrated in Fig. 3.

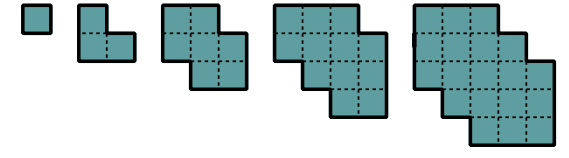

Fig. 3. The unique FAT $n$-ogons (symmetries excluded), for $n=4,6,8,10,12$.

Clearly, each piece r-piece is defined by four vertices. Each vertex is either in $\operatorname{INT}(P)$ (internal vertex) or is in $\operatorname{BND}(P)$ (boundary vertex). Similar definitions hold for edges. An edge $e$ of r-piece $R$ is called an internal edge if $e \cap \operatorname{INT}(P) \neq \emptyset$, and it is called boundary edge otherwise.

Lemma 8 The total number $\left|V_{i}\right|$ of internal vertices in $\Pi(P)$, when the grid $n$-ogon $P$ is as illustrated in Fig. 3 is given by (1)

$\left|V_{i}\right|= \begin{cases}\frac{3 r^{2}-2 r}{4}, & \text { for } r \text { even } \\ \frac{(3 r+1)(r-1)}{4}, & \text { for } r \text { odd }\end{cases}$

where $r$ is the number of reflex vertices of $P$.

PROOF. In case $r$ is even,

$$
\left|V_{i}\right|=2 \sum_{k=1}^{\frac{r}{2}}(r-k)
$$

and in case $r$ is odd

$$
\left|V_{i}\right|=\left(r-\frac{r+1}{2}\right)+2 \sum_{k=1}^{\frac{r-1}{2}}(r-k)
$$

Proposition 9 Every n-vertex orthogonal polygon $P$ such that the number of internal vertices of $\Pi(P)$ is given by (1) has at most a single reflex vertex in each horizontal and vertical line.

PROOF. We shall suppose first that $P$ is a grid $n$-ogon. Then, let $v_{L_{1}}=\left(x_{L_{1}}, y_{L_{1}}\right)$ and $v_{R_{1}}=$ $\left(x_{R_{1}}, y_{R_{1}}\right)$ be leftmost and rightmost reflex vertices of $P$, respectively. The horizontal chord with origin at $v_{L_{1}}$ can intersect at most $x_{R_{1}}-x_{L_{1}}$ vertical chords, since we shall not count the intersection with the vertical chord defined by $v_{L_{1}}$. The same may be said about the the horizontal chord with origin at $v_{R_{1}}$. There are exactly $r$ vertical and $r$ horizontal chords, and thus $x_{R_{1}}-x_{L_{1}} \leq r-1$. If there were $c$ vertical edges such that both extreme points are reflex vertices then $x_{R_{1}}-x_{L_{1}} \leq r-1-c$. 
This would imply that the number of internal vertices of $\Pi(P)$ would be strictly smaller than the value defined by (1). Indeed, we could proceed to consider the second leftmost vertex (for $x>x_{L_{1}}$ ), say $v_{L_{2}}$, then the second rightmost vertex (with $x<x_{R_{1}}$ ) and so forth. The horizontal chord that $v_{L_{2}}$ defines either intersects only the vertical chord defined by $v_{L_{1}}$ or it does not intersect it at all. So, it intersects at most $r-2-c$ vertical chords. In sum, $c$ should be null, and by symmetry, we would conclude that there is exactly a reflex vertex in each vertical grid line (for $x>1$ and $x<\frac{n}{2}=r+2$ ).

Now, if $P$ is not a grid $n$-ogon but is in general position, then $\Pi(P)$ has the same combinatorial structure as $\Pi(G R I D(P))$, so that we do not have to prove anything more.

If $P$ is not in general position, then let we render it in general position by a sufficiently small $\epsilon$-perturbation, so that the partition of this latter polygon would not have less internal vertices than $\Pi(P)$.

Corollary 10 For all grid n-ogons $P$, the number of internal vertices of $\Pi(P)$ is less than or equal to the value established by (1).

PROOF. It results from the proof of Proposition 9.

Theorem 11 Let $P$ be a grid $n$-ogon, $r=\frac{n-4}{2}$ the number of its reflex vertices. If $P$ is FAT then

$$
|\Pi(P)|=\left\{\begin{array}{cl}
\frac{3 r^{2}+6 r+4}{4}, & \text { for } r \text { even } \\
\frac{3(r+1)^{2}}{4}, & \text { for } r \text { odd }
\end{array}\right.
$$

and if $P$ is THIN then $|\Pi(P)|=2 r+1$.

PROOF. Suppose that $P$ is a grid $n$-ogon. Let $V, E$ and $F$ be the sets of all vertices, edges and faces of $\Pi(P)$, respectively. Let us denote by $V_{i}$ and $V_{b}$ the sets of all internal and boundary vertices of the pieces of $\Pi(P)$. Similarly, $E_{i}$ and $E_{b}$ represent the sets of all internal and boundary edges of such pieces. Then, $V=V_{i} \cup V_{b}$ and $E=E_{i} \cup E_{b}$. Being $P$ in general position, each chord we draw to form $\Pi(P)$ hits $\operatorname{BND}(P)$ in the interior of an edge and no two chords hit $\operatorname{BND}(P)$ in the same point. Hence, using O'Rourke's formula [2] we obtain $\left|E_{b}\right|=\left|V_{b}\right|=(2 r+4)+2 r=4 r+4$. It is easily seen that to obtain a FAT $n$-ogon we must maximize the number of internal vertices.

By Corollary 10,

$$
\max _{P}\left|V_{i}\right|= \begin{cases}\frac{3 r^{2}-2 r}{4}, & \text { for } r \text { even } \\ \frac{(3 r+1)(r-1)}{4}, & \text { for } r \text { odd }\end{cases}
$$

and, therefore, $\max _{P}|V|=\max _{P}\left(\left|V_{i}\right|+\left|V_{b}\right|\right)$ is given by

$$
\max _{P}|V|=\left\{\begin{array}{l}
\frac{3 r^{2}+14 r+16}{4}, \text { for } r \text { even } \\
\frac{3 r^{2}+14 r+15}{4}, \text { for } r \text { odd }
\end{array}\right.
$$

From Graph Theory [1] we know that the sum of the degrees of vertices in a graph is twice the number of its edges, that is, $\sum_{v \in V} \delta(v)=2|E|$. Using the definitions of grid $n$-ogon and of $\Pi(P)$, we may partition $V$ as

$$
V=V_{c} \cup V_{r} \cup\left(V_{b} \backslash\left(V_{c} \cup V_{r}\right)\right) \cup V_{i}
$$

$V_{r}$ and $V_{c}$ representing the sets of reflex and of convex vertices of $P$, respectively. Moreover, we may conclude that $\delta(v)=4$ for all $v \in V_{r} \cup V_{i}$, $\delta(v)=3$ for all $v \in V_{b} \backslash\left(V_{c} \cup V_{r}\right)$ and $\delta(v)=2$ for all $v \in V_{c}$. Hence,

$$
\begin{aligned}
2|E| & =\sum_{v \in V_{r} \cup V_{i}} \delta(v)+\sum_{v \in V_{c}} \delta(v)+\sum_{v \in V_{b} \backslash\left(V_{c} \cup V_{r}\right)} \delta(v) \\
& =4\left|V_{i}\right|+4\left|V_{r}\right|+2\left|V_{c}\right|+3\left(\left|V_{b}\right|-\left|V_{r}\right|-\left|V_{c}\right|\right) \\
& =4\left|V_{i}\right|+12 r+8
\end{aligned}
$$

and, consequently, $|E|=2\left|V_{i}\right|+6 r+4$.

Similarly, to obtain THIN $n$-ogons we must minimize the number of internal vertices of the arrangement. For all $n$, there are grid $n$-ogons such that $\left|V_{i}\right|=0$. Thus, for THIN $n$-ogons $|V|=4 r+4$.

Finally, to conclude the proof, we have to deduce the expression of the upper and lower bound of the number of faces of $\Pi(P)$, that is of $|\Pi(P)|$. Using Euler's formula $|F|=1+|E|-|V|$, and the expressions deduced above, we have $\max _{P}|F|=$ $1+2\left(\max _{P}\left|V_{i}\right|\right)+6 r+4-\max _{P}|V|$. That is, $\max _{P}|F|=\max _{P}\left|V_{i}\right|+6 r+5$, so that

$$
\max _{P}|F|=\left\{\begin{array}{c}
\frac{3 r^{2}+6 r+4}{4}, \text { for } r \text { even } \\
\frac{3(r+1)^{2}}{4}, \text { for } r \text { odd }
\end{array}\right.
$$

and 


\section{0th European Workshop on Computational Geometry}

$$
\begin{aligned}
\min _{P}|F| & =1+2\left(\min _{P}\left|V_{i}\right|\right)+6 r+4-\min _{P}|V| \\
& =1+6 r+4-4 r-4=2 r+1
\end{aligned}
$$

The existence of FAT grid $n$-ogons and THIN grid $n$-ogons for all $n$ (such that $n$ is even and $n \geq 4$ ) follows from Lemma 8 and the construction indicated in Fig. 5, respectively.

Figs. 4 and 5 show some THIN $n$-ogons.

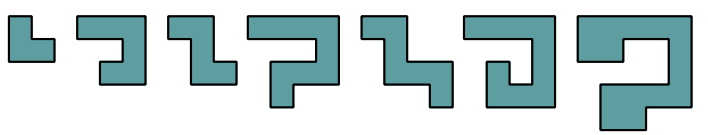

Fig. 4. Some grid $n$-gons with $\left|V_{i}\right|=0$.

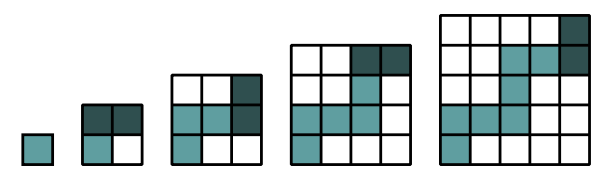

Fig. 5. Constructing the grid ogons of the smallest area, for $r=0,1,2,3,4, \ldots$ The area is $2 r+1$.

Based on the proof of Proposition 9, we may prove the uniqueness of FATs and fully characterize them.

Proposition 12 There is a single FAT n-ogon (except for symmetries of the grid) and its form is illustrated in Fig. 3.

PROOF. We saw that FAT $n$-ogons must have a single reflex vertex in each vertical grid-line, for $x>1$ and $x<\frac{n}{2}$. Also, the horizontal chords with origins at the reflex vertices that have $x=2$ and $x=\frac{n}{2}-1=r+1$, determine $2(r-1)$ internal points (by intersections with vertical chords). To achieve this value, they must be positioned as illustrated below on the left.


Moreover, the reflex vertices on the vertical gridlines $x=3$ and $x=r$ add $2(r-2)$ internal points. To achieve that, we may conclude by some simple case reasoning, that $v_{L_{2}}$ must be below $v_{L_{1}}$ and $v_{R_{2}}$ must be above $v_{R_{1}}$, as shown above on the right. And, so forth...

The area $A(P)$ of a grid $n$-ogon $P$ is the number of grid cells in its interior. FATs are not the grid $n$-ogons that have the largest area, except for small values of $n$, as we may see in Fig 6 .
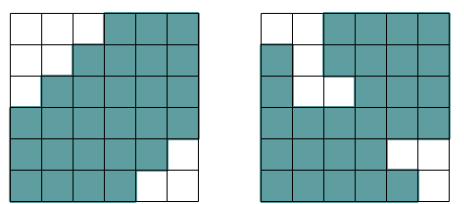

Fig. 6. On the left we see the FAT grid 14-ogon. It has area 27, whereas the grid 14-ogon on the right has area 28 , which is the maximum.

Proposition 13 Let $P$ be any grid n-ogon with $n \geq 8$ and $r$ reflex vertices $\left(r=\frac{n-4}{2}\right.$, for all $\left.P\right)$. Then

$$
2 r+1 \leq A(P) \leq r^{2}+3
$$

and there exist grid $n$-ogons having area $2 r+1$ (indeed, a single one except for symmetries) and grid $n$-ogons having area $r^{2}+3$.

PROOF. Our proof is strongly based on the INFLATE-PASTE method for generating grid ogons, that will be presented also at this workshop [3].

\section{References}

[1] Bondy, J., Murty, U.: Graph Theory with Applications. Elseiver Science, New York, (1976).

[2] O'Rourke, J.: An alternate proof of the rectilinear art gallery theorem. J. of Geometry 21 (1983) 118-130.

[3] Tomás, A. P., Bajuelos, A. L.: Quadratic-time linearspace algorithms for generating orthogonal polygons with a given number of vertices. (Extended abstract) In Proceedings European Workshop on Computational Geometry (2004). (This book)

[4] Tomás, A. P., Bajuelos, A. L., Marques, F.: Approximation algorithms to minimum vertex cover problems on polygons and terrains. In P.M.A Sloot et al. (Eds): Proc. of ICCS 2003, LNCS 2657, SpringerVerlag (2003) 869-878. 